\title{
Application de la méthode de relaxation à l'étude de quelques écoulements en milieu poreux
}

\section{Use of relaxation method for investigation of some flows in porous medium}

\author{
PAR G. MATTA \\ INGÉNIEUR DE L'ÉCOLE SUPÉRIEURE DE BEYROUTH \\ LICENCM L̇ SCIENGES
}

\begin{abstract}
L'équation aux dèrivés partielles de l'ćcoulement plan en milion hétérogène et anisotrope est traduite sous forme de differences finies. Les formules classiques relatives à un milieu homogène et isotrope en sont deduites comme cas particulier.

bans l'etude des conditions anx limites, la surface libre est supposée formée de segments de droites coincidant avec les lignes au quadrillage. Une fois les potentiels définitifs calculés aux nouds voisins de la surface libre, les points de celle-ci sont déterminés par interpolation ou extrapolation.
\end{abstract}

La methode ainsi rendue plus rapide est appliquée à l'étude de quelques éconlements plans. Les avantages de cette méthode théorique sur les analogies éfectriques sont mis en évidenee.
The differential equation for plane flow in at heterogeneous and anisolropie medinm is transposed in terms of finite differences. The classio formule concerned with a heterogeneous and antsotropic medium are deduced as a particular case.

In the investigation of boundary conditions, the free surface is assumed to be made up of straight segments coincident wilh the squming lines.

Once the definitive potentials have been calchlated for the nodes near the free surface, the points of these are determined by interpolation or extrapolation.

The method, thus speeded up, is applied to some plane floms. The advantages of this theorelical method ober electronic computers are poinfed ont.

\section{I. - INTRODUCTION}

L'étude mathématique des écoulements en milieu poreux à partir des transformations conformes est impossible dans la plupart des cas. Même dans le cas le plus simple d'un milieu homogène et isotrope où l'écoulement dérive d'un potentiel harmonique, les possibilités analytiques sont limitées.

On comprend donc bien l'intérêt des chercheurs de recourir à des solutions numériques remplaçant les dérivées partielles par des différences finies. La méthode de relaxation imaginée dans ce but par Southwell [1] a été appliquée à différents problèmes techniques et physiques. La méthode analogique des réseaux de conductances $[2,3$ et 4$]$ trouve ici ses bases théoriques.
Nous nous bornerons dans ce rapport à l'étude, par la méthode de relaxation, de certains écoulements à travers les barrages en terre. Nous mettrons en évidence la puissance de cette méthode à traiter tous les problèmes d'écoulements plans.

\section{Position du problème.}

Le problème à résoudre est le suivant : élant donné un champ o linćaire satisfaisant à une équation aux dérivées partielles de la forme:

$\mathrm{A} \frac{\partial^{2} \varphi}{\partial x^{2}}+\mathrm{B} \frac{\partial^{2} \varphi}{\partial y^{2}}+\mathrm{C} \frac{\partial^{2} \varphi}{\partial x \partial y}+\mathrm{D} \frac{\partial \vartheta}{\partial x}+\mathrm{E} \frac{\partial \varphi}{\partial y}+\mathrm{F} \varphi+\mathbf{C}=0$ 
trouver o à l'intérieur d'un domaine $D$ connaissant sur le contour de $D$ les valeurs de ou des expressions la renfermant.

Pour avoir la solution, on commence par convrir le domaine $\mathrm{D}$ d'un quadrillage et l'on alfecte aux différents nouds des valeurs de $p$ qu'on se propose d'améliorer par approximations successives. Pour ceci, on calcule pour chaque noud la valeur de $\varphi$ amélioré en remplaçant les dérivées partielles de (1) par des différences finies, comme il sera indiqué ci-après; on répète cette opération autant de fois qu'il le faut pour avoir une précision convenable.

\section{II. - EXPRESSION DES DÉRIVÉES PARTIELLES SOUS FORME DE DIFFÉRENCES FINIES CAS GÉNÉRAL D'UN MILIEU HÉTÉROGÈNE ET ANISOTROPE}

L'écoulement plan en milieu hétérogène et anisotrope satisfait à l'équation :

$$
\frac{\partial}{\partial x}\left[\mathbf{K}_{x} \cdot \frac{\partial v}{\partial x}\right]+\frac{\partial}{\partial z}\left[K_{z} \frac{\partial \omega}{\partial z}\right]=0
$$

dans laquelle :

p est la fonction de potentiel en un point du domaine étudié,

$\mathrm{K}_{x}$ et $\mathrm{K}_{z}$ sont les perméabilités principales dirigées suivant deux directions bien déterminées.

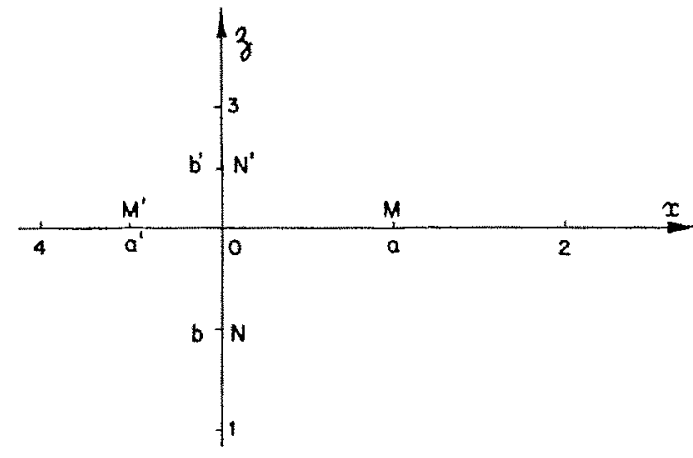

Fic. 1.

Si nous nous contentons d'une approximation du deuxième orỏre, l'équation (2) peut s'écrire, après des calculs simples et en adoptant les notations de la figure 1:

$$
\begin{aligned}
& \left\{\frac{1}{\left(a+a^{\prime}\right)^{2}}\left[\frac{a^{\prime 2}}{a^{2}}\left(\mathbf{K}_{x 2}-\mathbf{K}_{x 0}\right)-\left(\mathbf{K}_{n 4}-\mathbf{K}_{x 0}\right)\right]+\left.\frac{2}{a\left(a+a^{\prime}\right)} \mathbf{K}_{x 0}\right|_{1} ^{\prime}\left(\varphi_{2}-\varphi_{0}\right)\right. \\
& +\left\{-\frac{1}{\left(a+a^{\prime}\right)^{2}}\left[\left(\mathrm{~K}_{x 2}-\mathrm{K}_{x \rightarrow 0}\right)-\frac{a^{2}}{a^{\prime 2}}\left(\mathbf{K}_{x 4}-\mathrm{K}_{x 0}\right)_{0}\right]+\frac{2}{a^{\prime}\left(a+a^{\prime}\right)} \mathbf{K}_{x+0}\right\}\left(\varphi_{4}-\varphi_{0}\right) \\
& +\left\{\frac{1}{\left(b+b^{\prime}\right)^{2}}\left[\frac{b^{2}}{b^{2}}\left(\mathrm{~K}_{\mathrm{z1}}-\mathrm{K}_{z 0}\right)-\left(\mathrm{K}_{z 3}-\mathrm{K}_{z 0}\right)\right]+\frac{2}{b\left(b+b^{\prime}\right)} \mathrm{K}_{z 0}\right\}\left(\varphi_{1}-\varphi_{0}\right) \\
& +\left\{-\frac{1}{\left(b+b^{\prime}\right)^{2}}\left[\left(\mathbf{K}_{z 1}-\mathbf{K}_{z 0}\right)-\frac{b^{2}}{b^{\prime 2}}\left(\mathbf{K}_{z 3}-\mathbf{K}_{z 0}\right)\right]+\frac{2}{b^{\prime}\left(b+b^{\prime}\right)} \mathbf{K}_{z 0}\right\}\left(\varphi_{3}-\varphi_{0}\right)=0
\end{aligned}
$$

Si les mailles sont rectangulaires $\left(a=a^{\prime}\right.$ et $\left.b=b^{\prime}\right)$, l'équation (3) s'écrit :

$$
b^{2} / a^{2}\left[\mathbf{K}_{x \mathrm{M}}\left(\varphi_{2}-\varphi_{0}\right)+\mathbf{K}_{x \mathrm{M}}\left(\varphi_{4}-\varphi_{0}\right)\right]+\left[\mathbf{K}_{x \mathrm{~N}}\left(\varphi_{1}-\varphi_{0}\right)+\mathbf{K}_{i \mathrm{~N}} \cdot\left(\varphi_{3}-\varphi_{0}\right)\right]=0
$$

en appelant $M, M^{\prime}, N$ et $N^{\prime}$ les milieux respectifs des segments $(0,2)(0,4)(0,1)$ et $(0,3)$.

De ces formules générales, nous déduisons des formules très simples applicables à tous les écoulements plans ou tridimensionnels qui intéressent la pratique.

\section{III. - CONDITIONS AUX LIMITES}

\section{1. - Cas d'un milieu homogène et isotrope.}

Dans les écoulements de filtration en milieu homogène et isotrope, les frontières sont formées par des équipotentielles $\varphi=C^{i e}$ des lignes de suintement $\varphi=Z$ et des lignes de courant $\partial \varphi / \partial n=0$. Ces dernières peuvent avoir des positions inconnues a priori. 


\section{Lignes dE COURANT DE POSITION CONNUE.}

C'est le cas du fond imperméable et de certaines portions des parois latérales si l'effet de la capillarité n'est pas éliminé.

Pour éviter les étoiles irrégulières, nous faisons coincider ces lignes avec celles du quadrillage. Les potentiels aux nœuds qui y sont situés sont calculés en appliquant le principe de prolongement par symétrie.

D'après les notations de la figure 2, on a :

$$
4 \varphi_{0}=\varphi_{2}+\varphi_{4}+2 \varphi_{3}
$$

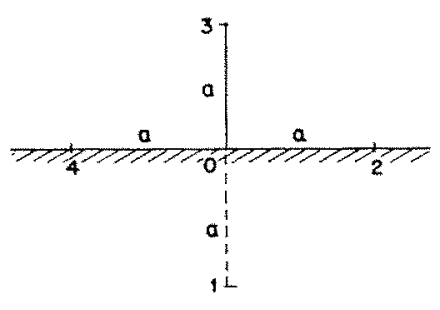

Fla. 2.

Ligne de COURANT DE POSITION INCONNUE, DÉTERMINATION DE LA SURFACE LIBRE.

La surface libre est une ligne de courant de position inconnue, elle vérifie à la fois les deux conditions :

$\frac{\partial \varphi}{\partial n}=0$ et $\varphi=Z$, ou plus généralemenl $\varphi=\left(Z+h_{c}\right)$

( $h$ c étant la pression capillaire qui règne sur la surface libre suivant les conditions de l'expérience).

Il existe plusieurs méthodes pour la détermination de cette surface, elles reviennent toutes $\dot{a}$ se donner a priori une position approchée de la ligne inconnue et à l'améliorer progressivement.

\section{Méthode A}

On impose à tous les, points du quadrillage situés sur la surface libre des potentiels vérifiant la condition $\varphi=Z+h_{c}$. Puis on améliore tous les potentiels aux nouds en utilisant, pour ceux qui sont situés au voisinage de la surface libre, la formule relative aux étoiles irrégulières. Une fois les valeurs nodales finales obtenues, on trace les équipotentielles et on déplace correctement la surface libre de facon qu'elle leur soit perpendiculaire. A partir de nouvelles conditions aux limites on recommence la même opération pour aboutir en fin de compte à la ligne définitive.

Comme on le voit, la condition $\partial \varphi / \partial n=0$ est considérée comme le critère définitif de l'exactitude du tracé.

\section{Méthode $B$}

On impose à chaque noeud voisin de la surface libre un potentiel donné par la formule: $p=Z+h_{0}$ dans laquelle $Z$ est égal à la cote du pied de la perpendiculaire abaissée du point considéré à la surface.

Ceci étant, on commence par corriger les potentiels à partir du fond imperméable en imposant la condition $\Delta^{2} \varphi=0$. Arrivé près de la surface libre, on déplace celle-ci convenablement de facon à ce que les potentiels calculés aux nœuds voisins soient égaux à ceux qu'on s'étail fixć.

Pour simplifier cette méthode, on peut prolonger les mailles au-delà de la surface libre et affecter chaque nœud fictif ainsi formé d'un potentiel déterminé, comme il est indiqué ci-dessus. Ainsi, on aura affaire à des ćtoiles régulières bien plus faciles à traiter.

Malgré tout, ces mélhodes de relaxation qui déterminent la surface libre par des tâtonnements successifs, exigent des calculs longs, incompatibles avec un usage fréquent el systématique. Ces tâtonnements rendent d'ailleurs les méthodes moins rapides, il faudrait les éliminer.

\section{Méthode proposée $C$}

Au lieu de partir d'une position approchée de la surface libre, nous considirons celle-ci comme formée de segments de droiles coincidant avec les lignes du quadrillage. Puis, en utilisant la formule (4) ćtablie pour les lignes de courant de position connue, nous corrigeons par approximations successives les potentiels aux nouds situés sur ces mailles fronticres.

Une fois les valeurs nodales définitives oblenues, nous déduisons par extrapolation ou interpolation les points appartenant a la ligne libre exacte qui vérifient $\varphi=Z+h_{i}$.

Il nous est apparu qu'en partant de deux fronlieres différentes nous aboutissons à deux surfaces libres s'écartant dans des proportions par fois appréciables. Comme dans la premiere méthode, nous considérons que l'orthogonalité des equipotentielles el de la surface libre est le critere de l'exactitude de la solution. Il semble donc que, la aussi, nous sommes assujellis à de longs tâtonnements.

Les applications montrent que cetle orthogonalité est obtenue du premier coup en partant d'une frontière fictive supéricure à la ligne libre réelle. C'est d'ailleurs le cas ou les résultats concordent, avec une approximation tres satisfaisante, avec ceux que nous oblenons a partir d'un calcul plus rigoureux par la méthode générale. La figure 3 donne une comparaison entre la surface libre déterminće par la méthode $a$ ) et celle qui est calculée par la méthode proposéc. L'ecart 
entre les deux lignes, mesuré suivant la verticale et rapporté à la cote du point considéré au-dessus du fond imperméable, ne dépasse pas $1 \%$.

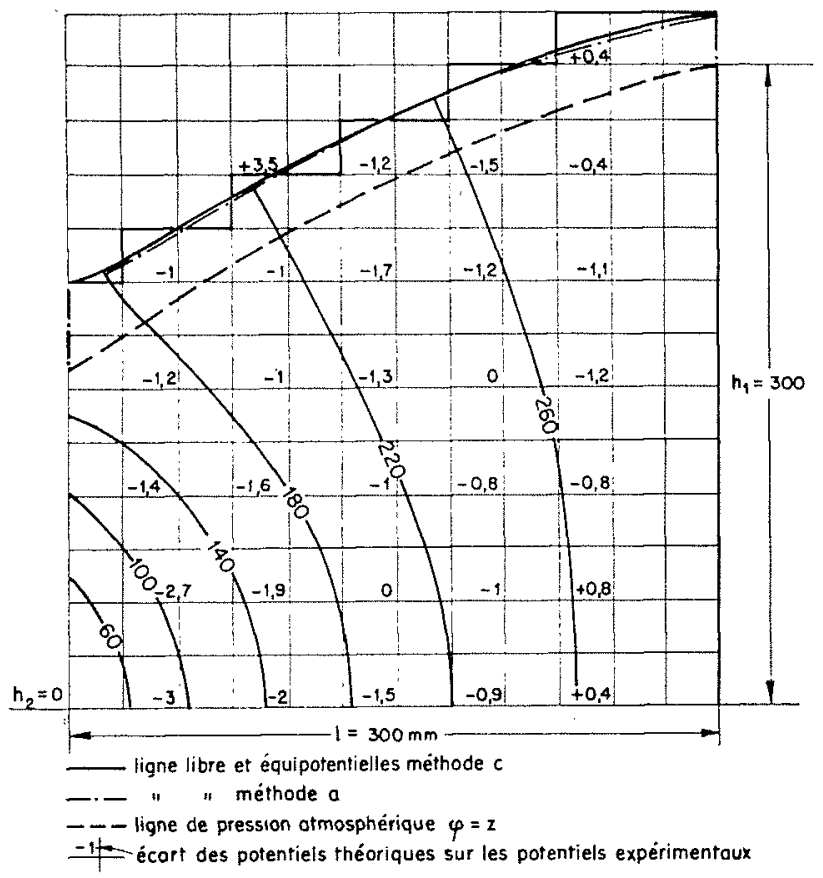

Fig. 3.

\section{2. - Cas d'un milieu homogène anisotrope.}

Nous supposons que les perméabilités principales $\mathrm{K}_{l}$ et $\mathrm{K}_{v}$ sont horizontale et verticale et nous donnons aux lignes du quadrillage ces mêmes directions.

$\mathrm{Si} u$ et $v$ sont les composantes de la vitesse suivant ces directions, la loi de Darcy s'écrit :

$$
\begin{aligned}
& u=-K_{l l} \varphi_{s t}^{\prime} \\
& v=-K_{r} \varphi_{z}^{\prime}
\end{aligned}
$$

Ces équations montrent que le vecteur vitesse n'est pas co-linéaire au gradient de la fonction $\varphi$. La dérivée partielle $\partial_{\varphi} / \partial n$ est donc différente de zéro. Les particularités introduites par l'anisotropie concernent tout simplement les lignes de courant : fond imperméable et surface libre.

\section{- Fond imperméable :}

Le fond imperméable étant supposé horizontal, le gradient de $\varphi$ est co-linéaire avec la vitesse le long de cette frontière. On a, comme dans un milieu isotrope $: \partial \varphi / \partial n=0$.

En disposant les mailles de façon à ce que le fond imperméable soit confondu avec une ligne du quadrillage (figure 2), on a :

$$
\varphi_{2}+\varphi_{4}-2 \varphi_{0}+2\left(K_{v} / K_{h}\right)\left(\varphi_{3}-\varphi_{0}\right)=0
$$

\section{- Surface libre :}

En un point de la surface libre, la vitesse fait avec le gradient du potentiel un angle $\omega^{\prime}$ donné par la formule :

$$
\operatorname{tg} \omega^{\prime}=\frac{\left(\mathbf{K}_{v}-\mathbf{K}_{h}\right) \operatorname{tg} 0}{\mathbf{K}_{r}+\mathbf{K}_{h} \operatorname{tg}^{2} \theta}
$$

dans laquelle $\theta$ est l'angle de la vitesse avec l'horizontale.

Si l'on détermine la surface libre par approximations successives à partir des méthodes (A) et (B) données pour les massifs homogènes et isotropes, il suffit de remplacer la condition d'orthogonalité des équipotentielles et surface libre par la condition d'intersection sous un angle $\left[(\pi / 2)-\omega^{\prime}\right]$, l'angle $\omega^{\prime}$ étant variable d'un point à un autre.

Par contre, la méthode proposée est utilisée sans modification. En effet, si l'on suppose la surface libre formée de brins horizontaux et verticaux, $\omega^{\prime}$ serait nul et les formules d'approximation du type (3) pourraient être utilisées.

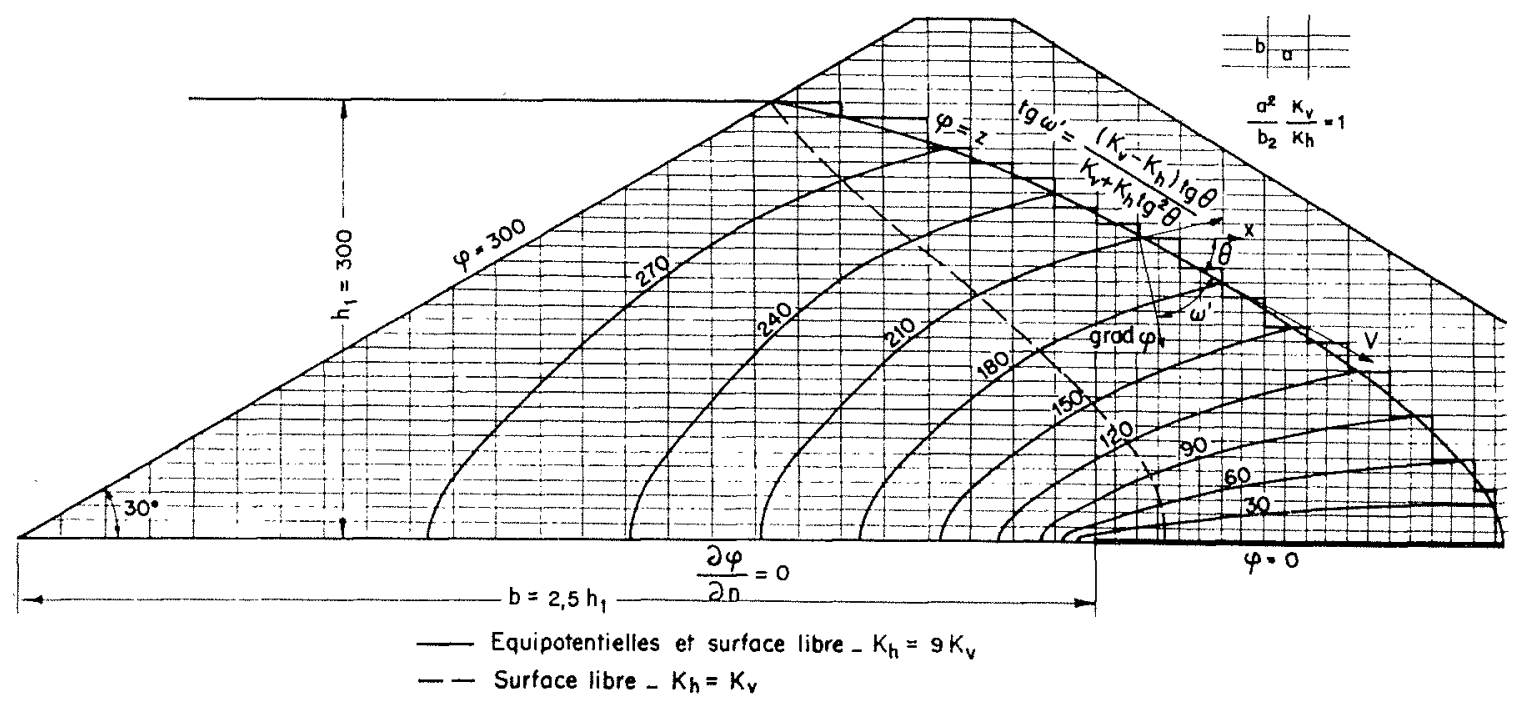

FIG. 4. 
Nous traçons sur la figure 4 le réseau d'écoulement vers un drain situé à l'aval dans une digue anisotrope.

\section{3. - Cas d'un massif zoné.}

A part les conditions aux limites qui se présentent dans les digues homogènes et isotropes, la surface de séparation de deux massifs de perméabilités différentes constitue une frontière supplémentaire.

Déterminons les potentiels aux nœuds voisins de cette frontière. Trois cas sont à distinguer :

- Cas ou la surface de séparation ne passe que par des nouds (fig. 5).

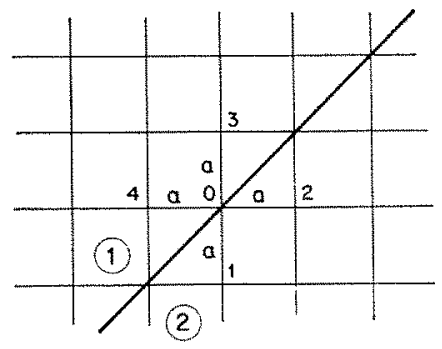

F1G. 5 .

En appliquant la formule générale (5), on obtient :

$$
K_{2}\left(\varphi_{1}+\varphi_{2}-2 \varphi_{0}\right)+K_{1}\left(\varphi_{3}+\varphi_{4}-2 \varphi_{0}\right)=0
$$

qui s'écrit encore :

$$
2\left(1+\frac{K_{2}}{K_{1}}\right) \varphi_{0}=\varphi_{1}+\varphi_{2}+\varphi_{3}+\varphi_{4}
$$

où $K_{1}$ et $K_{2}$ sont les perméabilités respectives des zones 1 et 2 - cas où la surface de séparation est confondue avec une limite du quadrillage (fig. 6).

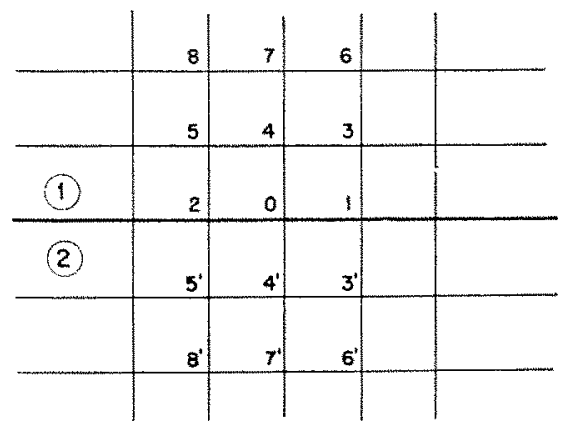

FIG. 6.

Les conditions de continuité du potentiel (5) au point $O$ peuvent s'écrire :

$$
\left(\varphi_{0}\right)_{1}=\left(\varphi_{0}\right)_{2} \quad \text { et } \quad K_{1}\left(\frac{\partial \varphi_{1}}{\partial n}\right)_{0}=K_{2}\left(\frac{\partial \varphi_{n}}{\partial n}\right)_{0}
$$

ou, sous forme de différences finies :

$3\left(1+\frac{K_{2}}{K_{1}}\right) \varphi_{0}=\left(4 \varphi_{4}-\varphi_{7}\right)+\frac{K_{2}}{K_{1}}\left(4 \varphi_{4}^{\prime}-\varphi_{7}^{\prime}\right)$

On a, d'autre part:

$$
4 \varphi_{4}=\varphi_{0}+\varphi_{3}+\varphi_{5}+\varphi_{i}
$$

et :

$$
4 \varphi_{4}=\varphi_{0}+\varphi_{3}+\varphi_{5}+\varphi_{7}
$$

L'équation (6) s'écrit donc :

$$
2\left(1+\frac{K_{2}}{K_{1}}\right) \varphi_{0}=\left(\varphi_{3}+\varphi_{9}\right)+\frac{K_{2}}{K_{1}}\left(\varphi_{3}+\varphi_{5}\right)
$$

Pour trouver une formule en $\varphi_{1}, \varphi_{2}, \varphi_{4}$ et $\varphi_{4}$, on écrit la formule (16) pour les points 1 et 2 On obtient finalement :

$$
4\left(1+\frac{K_{2}}{K_{1}}\right) \varphi_{0}=2 \varphi_{4}+\varphi_{1}+\varphi_{2}+\frac{K_{2}}{K_{1}}\left(2 \varphi_{4}+\varphi_{1}+\varphi_{2}\right)
$$

Si l'on fait $K_{1}=K_{2}$, on retrouve la formule elassique :

$$
4 \varphi_{0}=\varphi_{1}+\varphi_{2}+\varphi_{4}+\varphi_{4}
$$

- cas général d'un plan de séparation quelconque.

La méthode la plus simple consiste à utiliser des étoiles rectangulaires de telle sorte que la surface de séparation ne passe que par des nouds (fig. 7).

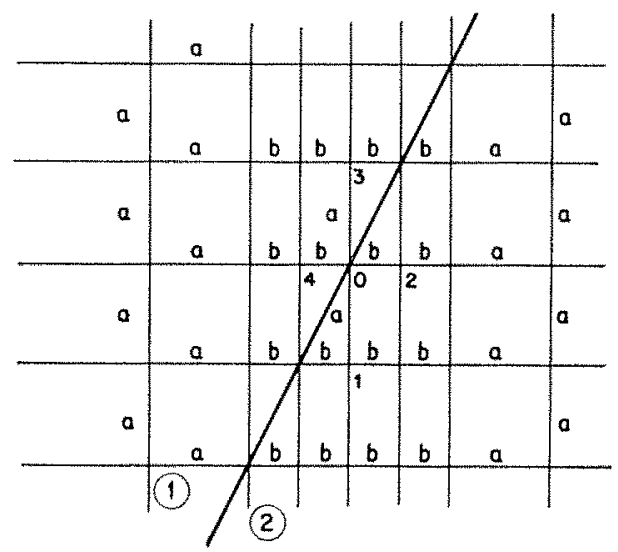

FIG. 7 .

En appliquant la formule générale (5) au noud $O$ par exemple et en supposant un degré d'anisotropie différent dans les deux zores, il vient :

$$
\begin{aligned}
\mathrm{K}_{v 2} \times & \frac{1}{a_{2}}\left(\varphi_{1}-\varphi_{0}\right)+\mathrm{K}_{h 2} \frac{1}{b_{2}}\left(\varphi_{2}-\varphi_{0}\right) \\
& \quad+\mathrm{K}_{v 1} \frac{1}{a_{2}}\left(\varphi_{3}-\varphi_{0}\right)+\mathrm{K}_{h 1} \frac{1}{b_{3}}\left(\varphi_{4}-\varphi_{0}\right)=0
\end{aligned}
$$


ou encore :

$$
\begin{aligned}
& {\left[\mathbf{K}_{t^{2} 2}\left(\varphi_{1}-\varphi_{0}\right)+K_{01}\left(\varphi_{3}-\varphi_{0}\right)\right]} \\
& +\frac{a_{2}}{b_{2}}\left[K_{h 2}\left(\varphi_{2}-\varphi_{0}\right)+K_{h 1}\left(\varphi_{4}-\varphi_{0}\right)\right]=0
\end{aligned}
$$

Si la digue est isotrope: $\left(\mathbf{K}_{v 2}=\mathbf{K}_{f^{2} 2}\right.$ et $\left.\mathbf{K}_{z 1}=\mathbf{K}_{h 1}\right)$. on a :

$$
\begin{aligned}
& {\left[K_{2}\left(\varphi_{1}-\varphi_{0}\right)+K_{1}\left(\varphi_{3}-\varphi_{0}\right)\right]} \\
& +\frac{a_{2}}{b_{2}}\left[K_{2}\left(\varphi_{2}-\varphi_{0}\right)+K_{1}\left(\varphi_{4}-\varphi_{0}\right)\right]=0
\end{aligned}
$$

Dans ce dernier cas, la déviation des lignes de courant est donnée par la formule :

$$
K_{1} \operatorname{tg}\left(\theta_{1}-\gamma\right)=K_{2} \operatorname{tg}\left(\theta_{2}-\gamma\right) \quad(\text { fig. } 8)
$$

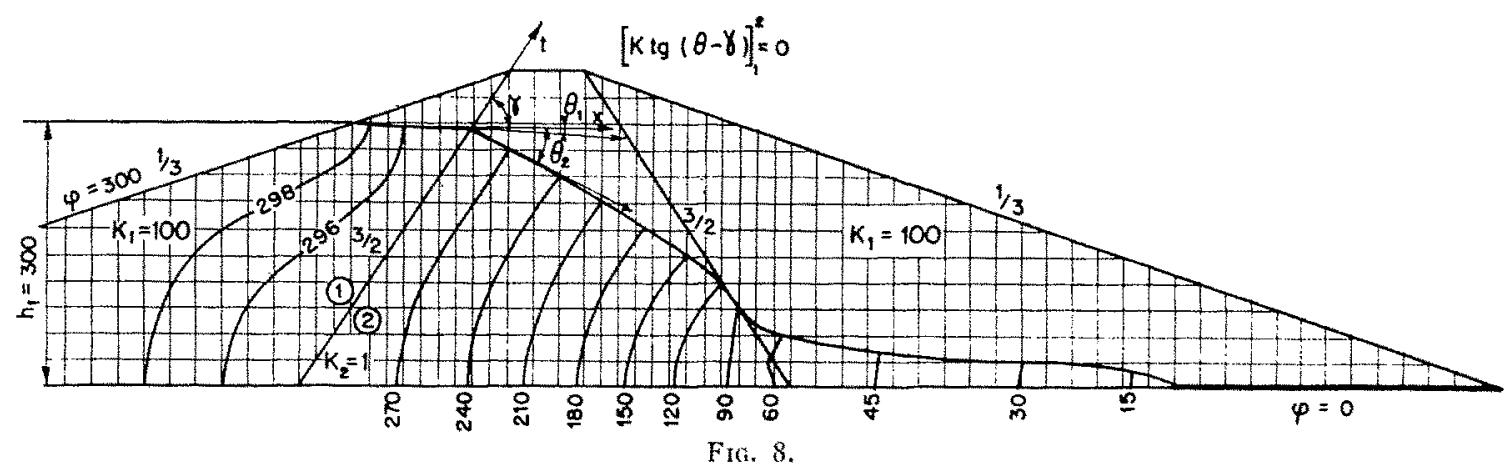

\section{IV. - CONCLUSIONS}

I1 ressort de cette étude que la méthode de relaxation est excellente pour résoudre tous les problèmes d'écoulements plans en milieu poreux. Moyennant quelques simplifications en ce qui concerne la détermination de la surface libre, la méthode est rendue plus rapide que celles qui sont basées sur les analogies électriques. Elle ne nécessite pas la construction de réseaux de conductances et ne fait done pas intervenir des erreurs autres que celles qui tiennent à l'approximation du calcul.

Les problemes tridimensionnels peurent être abordés mais nécessitent des développements plus importants dus au plus grand nombre de mailles intervenant dans le domaine étudié.

Les problèmes posés par le régime variable sont résolus avantageusement par la méthode des réseaux hydrauliques.

\section{BIBLIOGRAPHIE}

1. A.V. Sourhwets. - Relaxation methods in Engineering Science. Oxford Univ. Press. 1940.

2. D.C. DE PACKH. - A solution network for the approximate solution of the Laplace equations. Rev. Scient, instr. vol. 18-1947.

3. L. TASNY-Tschasswy. - The triangulation of a iwo dimensional continuum for the purpose of the appli- cation solution of the second order partial differm ential equation.

4. G. Lrebmanx. - Solution of partial differential cquations with a resistance net analogue. Brit. J. Appli. Phys, 1950.

5. E. Dunand. - Electrostatique et Magnétostatique. 6. P. Huard de la Marne. - Thèse, Paris 1956.

DI S CUS I I N

Président : M. Chapouthier

M. le Président remercie M. Matra d'avoir attiré l'attention sur le calcul par approximations successives dans un domaine où comme dans beaucoup d'autres, le calcul at l'experrience se prêtent un mutuel concours.

II. HaBm pose à M. MatTa la question ci-après:
"Dans son exposé, M. Matra a indiqué qu'on pouvait * aboutir a differentes lignes de saturation lorsqu'on « part de quadrillages initiaux différents, en particuliex " situés en dessous et au-dessus de la ligne de satu« ration. 
«Lorsqu'on utilise la méthode des analogies électri« ques pour la détermination d'un réseau d'écoulement, « il arrive, lorsque la ligne de saturation présente des « courbures de signes différenls, que la série convergente «qui permet d'atteindre la ligne de saturation défini« tive, soit une séric alternée. Un tel cas se produit-il « avec le calcul par relaxation et, dans l'affirmative, « est-ce qu'on aboutit à une ligne bien définie?»

M. MatTa répond que ses approximations ne comportent pas de séries alternées et que dans le cas où la ligne brisée d'approximation est inférieure à la ligne réelle supposce connue, les équipotentielles font des angles aigus avec la surface libre; tandis que, dans le cas où la ligne brisée est supérieure à la ligne réelle, les intersections de colles-ci avec les équipotentielles sont strictement à angle droit, pour autant que l'écart entre ligne réelle et ligne approchée n'est pas trop sensible. Il est done préférable de partir d'une surface libre approchée légèrement supérieure à la surface réelle.

D'autre part, M. HABlB remarque que le calcul donné par M. Matra pour la détermination du réseau d'écoulement y compris la frange capillaire suppose le coefficient de perméabilité constant. En fait, la frange capillaire correspond à un milieu non saturé. Or, le coefficient do perméabilité des sols décroit très rapidement lorsque la teneur en air augmente (de 1 à $1 / 1000$ facilement). Dans ces conditions, le calcul présenté donne un débit d'infil. trations capillaires beaucoup trop important par rapport à ce qui se passe dans la nature.

M. MatrTa précise que le tracé du réseau des équipotentielles et des lignes de courant n'est pas tellement influencé par les variations du coefficient de perméabilité, et que le débit capillaire mesuré à partir du réseau sous forme de $Q / K$ concorde avec la valeur donnèe par l'expérience.

M. Huard de la Marne s'étonne que la comparaison de la méthode de relaxation avec la méthode des analogies électriques ait donné, d"après M. Matta, un écart de $3 \%$ au détriment de celle-ci qui, généralement, coïncide à quelques millièmes près avec les méthodes de calcul.

M. MatTa répond qu'il a comparé la methode ue relaxation, non à celle des réseaux de conductances, mais à celle des modèles en sable réalisés avec une technique spéciale et pense que l'erreur de $3 \%$ que l'on constate au voisinage de la zone capillaire est due au modèle expélimental partiellement saturé dans cette zone et qui ne donne pas par conséquent, des valeurs assez précises des charges.

Par ailleurs, M. MatTa a comparé sa méthode á celles de la cuve rhéographique et du papier conducteur : les divergences constatées sont plutôt dues au modèle électrique. Il estime d'autre part que la méthode de relaxation est plus précise que la méthode des réseaux de conductances, parce que celle-ci introduit, outre les erreurs d'expériences, les erreurs dues aux raleurs des résistances qui sont fonction de l'écartement des mailles.

M. Huanu de la Marre indique que la précision de la méthode des réseanx de conductances (qui n'est autre qu'une relaxation automatique) dépend essentiellement de l'imposition des conditions aux limites electriques, et très peu de la plécision des valeurs des résistances, ni des dimensions non faibles des mailles. Elle permet d'autre part de représenter exactement la forme de la surface libre (à $1 \%$ de la hauteur de charge, par exemple), mal. gré un maillage parfois assez grand et une plécision des résistances de $0,5 \%$. Aussi, cette méthode ne peut être moins précise que la méthode de relaxation où l'approxi. mation polygonale de la surface libre superpose à l'écoulement réel une cortaine épaisseur fictive de sable mouillé. Tìs exactement, d'après la théorie des différences finies, le fait de limiter le domaine de relaxalion suivant un contour polygonal revient à étudier l'écoulement de filtration en charge limité par une paroi imperméable passant par les sommets saillants du contour polygonal en question.

Enfin, les modèles hydrauliques en sable, bien que roprésentant des écoulements réels, ne peuvent ètre considérés comme des critères sûrs, leurs constitutions n'étant jamais connues avec précision.

De toutes façons, les précisions respectives des deux méthodes sont toutes denx amplement suffisantes pour les besoins pratiques; seules lenrs commodités d'utilisation et leurs rapidités peuvent étre comparées.

N. le Président conclut que la méthode analogique expérimentale et la méthode théorique de calcul ont peut-être toutes deux des progrès à faire, l'une aidant l'autre, pour arriver chacune à une plus grande précision.

Sur la demande de M. Huard de la Mante, M. Matta précise que la méthode de relaxation lui a demandé une dizaine d'heures pour résoudre, d'abord, le problème d'une digue à parois verticales, tandis qu'ensuite, l'habitude aidant, elle lui a permis de traiter en trois ou quatre heures le cas d'une digue a parois inclinées.

Sur la demande de M. Hupner, M. le Président et M. Matra donnent quelques précisions sur le sens et l'origine du mot «relaxation》, associé à cette méthode : ce mot signifie : «approximations successives» et d'autre part, donne l'idée d'un réseau maillé; cette dénomination, d'origine anglo-saxomne, a été donnée probablement par Soutliwell.

M. Bonnin suggère à M. Matra :

1. D'améliorer la précision du tracé de la surface libre lorsque sa pente est assez différente de $45^{\circ}$, en transformant la maille carrée en maille rectangulaire;

$2^{\circ}$ D’appliquer à la méthode la distorsion géométrique, en cas de perméabilité anisolrope, afin de conserver l'orthogonalité des lignes de courant et des équipotenticlles.

M. Matra pense que la premier procédé compliquerait un peu le problène; quant au deuxième, M. Matra l'a appliqué et a obtenu des résultats satisfaisants qu'il a comparés à ceux qu'il a obtenus à partir des mailles rectaugulaires. M. MATrA signale que l'avantage des mail les rectangulaires est de traiter les problèmes anisolropes avec la même précision et la même rapidité que les problemes isotropes. 